TRANSACTIONS OF THE

AMERICAN MATHEMATICAL SOCIETY

Volume 351, Number 11, Pages 4675-4692

S 0002-9947(99)02328-4

Article electronically published on July 20, 1999

\title{
DECOMPOSITION OF $B(G)$
}

\author{
TIANXUAN MIAO
}

\begin{abstract}
For any locally compact group $G$, let $A(G)$ and $B(G)$ be the Fourier and the Fourier-Stieltjes algebras of $G$, respectively. $B(G)$ is decomposed as a direct sum of $A(G)$ and $B^{s}(G)$, where $B^{s}(G)$ is a subspace of $B(G)$ consisting of all elements $b \in B(G)$ that satisfy the property: for any $\epsilon>0$ and any compact subset $K \subset G$, there is an $f \in L^{1}(G)$ with $\|f\|_{C^{*}(G)} \leq 1$ and $\operatorname{supp}(f) \subset K^{c}$ such that $|\langle f, b\rangle|>\|b\|-\epsilon . \quad A(G)$ is characterized by the following: an element $b \in B(G)$ is in $A(G)$ if and only if, for any $\epsilon>0$, there is a compact subset $K \subset G$ such that $|\langle f, b\rangle|<\epsilon$ for all $f \in L^{1}(G)$ with $\|f\|_{C^{*}(G)} \leq 1$ and $\operatorname{supp}(f) \subset K^{c}$. Note that we do not assume the amenability of $G$. Consequently, we have $\|1+a\|=1+\|a\|$ for all $a \in A(G)$ if $G$ is noncompact. We will apply this characterization of $B^{s}(G)$ to investigate the general properties of $B^{s}(G)$ and we will see that $B^{s}(G)$ is not a subalgebra of $B(G)$ even for abelian locally compact groups. If $G$ is an amenable locally compact group, then $B^{s}(G)$ is the subspace of $B(G)$ consisting of all elements $b \in B(G)$ with the property that for any compact subset $K \subseteq G$, $\|b\|=\sup \left\{\|a b\|: a \in A(G), \operatorname{supp}(a) \subseteq K^{c}\right.$ and $\left.\|a\| \leq 1\right\}$.
\end{abstract}

\section{INTRODUCTION}

For any locally compact topological group $G$, let $A(G)$ and $B(G)$ be the Fourier and the Fourier-Stieltjes algebras of $G$, respectively. If $G$ is abelian, then $G$ has the dual group $\hat{G}$, and $A(G) \simeq L^{1}(\hat{G})$ and $B(G) \simeq M(\hat{G})$, the space of all bounded measures on $\hat{G}$. By the Lebesgue Decomposition Theorem, $M(\hat{G})=L^{1}(\hat{G}) \oplus$ $M_{s}(\hat{G})$, where $M_{s}(\hat{G})$ is the subspace of $M(\hat{G})$ consisting of all the measures in $M(\hat{G})$ that are singular to the Haar measure on $\hat{G}$. If $G$ is an amenable locally compact group, Flory in [10] proved a Lebesgue-type decomposition theorem for $B(G)$ (also see Pier [19], p. 210). Amenability plays a key role in his proof. Arsac [1] showed that $B(G)$ is a direct sum of $A(G)$ and a subspace $B^{s}(G)$ of $B(G)$ for a general locally compact group. The main purpose of this paper is to study the properties of $A(G)$ and $B^{s}(G)$. It is well known that $A(G) \subseteq C_{0}(G) \cap B(G)$, but $A(G) \neq C_{0}(G) \cap B(G)$ even for some [AR] groups (see Baggett and Taylor [2], and also Taylor [21]). We characterize $A(G)$ by the following: an element $b \in B(G)$ is in $A(G)$ if and only if, for any $\epsilon>0$, there is a compact subset $K \subset G$ such that $|\langle f, b\rangle|<\epsilon$ for all $f \in L^{1}(G)$ with $\|f\|_{C^{*}(G)} \leq 1$ and $\operatorname{supp}(f) \subset K^{c}$. Note that we do not assume the amenability of $G$ (see Flory [9] for the case of amenable

Received by the editors April 29, 1997.

1991 Mathematics Subject Classification. Primary 43A07.

Key words and phrases. Locally compact groups, amenable groups, the Fourier algebra of a locally compact group, the Fourier-Stieltjes algebra of a locally compact group, the Lebesgue-type decomposition of the Fourier-Stieltjes algebra.

This research is supported by an NSERC grant. 
locally compact groups). We will prove in this paper that $B^{s}(G)$ is a subspace of $B(G)$ invariant under convolution by the elements of $M(G)$, consisting of all elements $b \in B(G)$ that satisfy the property: for any $\epsilon>0$ and any compact subset $K \subset G$, there is an $f \in L^{1}(G)$ with $\|f\|_{C^{*}(G)} \leq 1$ and $\operatorname{supp}(f) \subset K^{c}$ such that $|\langle f, b\rangle|>\|b\|-\epsilon$. Consequently, we have that $1 \in B^{s}(G)$ and so $\|1+a\|=1+\|a\|$ for all $a \in A(G)$ if $G$ is noncompact. It is well known that, when $G$ is amenable, $\|b\|=\sup \{\|a b\|: a \in A(G),\|a\| \leq 1\}$ for all $b \in B(G)$. We will also characterize $A(G)$ and $B^{s}(G)$ in terms of this multiplier norm condition on $B(G)$ for amenable locally compact groups. Then we will apply these characterizations of $B^{s}(G)$ to investigate the general properties of $B^{s}(G)$. We will show that, for instance, $b \in$ $B(G)$ is positive definite (or hermitian) if and only if $b$ can be decomposed into a direct sum of positive definite (or hermitian) elements $b_{u} \in A(G)$ and $b_{s} \in B^{s}(G)$. If $b \in B^{s}(G)$ is hermitian, then $b^{+}, b^{-}$and $|b|$ are also in $B^{s}(G)$, where $b=b^{+}-b^{-}$ is the Jordan decomposition of $b$. It is well known that $A(G)$ is a closed ideal of $B(G)$. We will see that $B^{s}(G)$ is not a subalgebra of $B(G)$ even for some abelian locally compact groups, and we will also show that $B^{s}(G)$ is invariant with respect to subgroups and quotient groups for some subgroups. The main ingredients of the proofs are the Arsac's decomposition theorem mentioned above and the following theorem.

Theorem 1.1. A bounded sequence $f_{n} \in C(\Omega)$ is weakly convergent to $f \in C(\Omega)$ if and only if $f_{n}$ is pointwise convergent to $f$, where $\Omega$ is a compact Hausdorff space and $C(\Omega)$ is the Banach space of all bounded continuous functions on $\Omega$ with the sup-norm (see Diestel [5], p. 66).

We organize this paper as follows. In section 2 , we collect the notations, definitions and some known results that will be used in this paper. In section 3 , we give characterizations of $A(G)$ and $B^{s}(G)$. These are the main results of this paper. In section 4 , we will study some properties of $B^{s}(G)$ by applying the characterization theorems proved in section 3 . In section 5 , we will apply this decomposition theorem to study the Dunford-Pettis property of $B_{\rho}(G)$ and to prove a result concerning the norm of $1+a$ for any $a \in A(G)$. In section 6 , we will discuss the case of $p \neq 2$.

\section{Preliminaries and some notations}

Let $G$ be a locally compact group with a fixed left Haar measure $\lambda$. If $G$ is compact, we assume $\lambda(G)=1$. For any $f: G \rightarrow C$, and $x \in G$, the left [right] translation of $f$ by $x$ is defined by ${ }_{x} f(y)=f(x y)\left[f_{x}(y)=f(y x)\right]$. The support of $f$ is denoted by $\operatorname{supp}(f)$. Let $L^{p}(G)$ be the associated Lebesgue spaces $(1 \leq p \leq \infty)$. We say that $G$ is amenable if there exists an $m \in L^{\infty}(G)^{*}$ such that $m \geq 0, m(1)=$ 1 and $m\left({ }_{x} f\right)=m(f)$ for all $x \in G$ and $f \in L^{\infty}(G)$. Properties of amenable groups can be found in Greenleaf [12], Paterson [18] and Pier [19].

We denote the modular function of $G$ by $\Delta$. For a subset $E$ of $G$, the characteristic function of $E$ is denoted by $1_{E}$ and the complement of $E$ in $G$ is denoted by $E^{c}=G \sim E$. Let $\Omega$ be any topological space. $C(\Omega)$ is as usual the Banach algebra of all continuous bounded functions on $\Omega$ with uniform norm and pointwise multiplication. The Dirac measure $\delta_{x}$ at $x \in \Omega$ is defined by $\left\langle\delta_{x}, f\right\rangle=f(x)$ for $f \in C(\Omega)$. If $X$ and $Y$ are Banach spaces in duality, $\sigma(X, Y)$ will denote as usual the weakest topology on $X$ which makes all linear functionals in $Y$ continuous. $X^{*}$ is the conjugate Banach space of $X$ and $\mathcal{B}(X)$ denotes the space of continuous linear operators from $X$ to $X$. If different norms will sometimes occur on $X$ we will write 
$\|\cdot\|_{X}$. Let $x \in X$ and $f \in X^{*}$, the value of $f$ at $x, f(x)$, is sometimes denoted by $\langle f, x\rangle$ or $\langle x, f\rangle$ in duality. If $H$ is a Hilbert space, $\langle x, y\rangle$ is the inner product of $x$ and $y$ in $H$. This notation does not cause any confusion since $H^{*}=H$ for a Hilbert space.

Let $G$ be a locally compact group with unit $e$. For any complex-valued function $f$ on $G$, we define as usual

$$
\check{f}(x)=f\left(x^{-1}\right), \tilde{f}(x)=\overline{f\left(x^{-1}\right)} \text { and } f^{*}(x)=\left(\frac{1}{\Delta(x)}\right) \tilde{f}(x), \quad x \in G .
$$

Let $A(G)$ and $B(G)$ be the Fourier and the Fourier-Stieltjes algebras of $G$, respectively (see Eymard [8] for $A(G)$ and $B(G)$ ). $A(G)$ consists of all functions on $G$ in the form of $f * \tilde{g}$ for $f, g \in L^{2}(G)$ and the norm of an element $a \in A(G)$ is given by

$$
\|a\|=\inf \left\{\|f\|_{2}\|g\|_{2}: a=f * \tilde{g}, f, g \in L^{2}(G)\right\} .
$$

Actually, this infimum can be attained and $A(G)$ has a bounded approximate identity if and only if $G$ is amenable. Occasionally, we will use the following fact: for any compact subset $K$ of $G$, there is an $a_{K} \in A(G)$ such that $a_{K}=1$ on $K$ and $\operatorname{supp}\left(a_{K}\right)$ is compact (see Pier [19], Theorem $10.4(\mathrm{a})$ ). If $G$ is amenable, this $a_{K}$ can be chosen in such a way that $\left\|a_{K}\right\|$ is arbitrarily close to 1 .

It is known that $B(G)$ is the linear span of the set $P(G)$ of continuous positive definite functions on $G$ and $B(G)$ is also the Banach space dual of the group $C^{*}$ algebra $C^{*}(G)$. An element $b \in B(G)$ is a positive functional on $C^{*}(G)$ if and only if $b$ is positive definite, and $b$ is a hermitian functional on $C^{*}(G)$ if and only if $b=\tilde{b}$ (see Eymard [8]).

Let $b \in B(G)$ be hermitian. Then there are unique positive definite functions $b^{+}$ and $b^{-}$such that $b=b^{+}-b^{-}$and $\|b\|=\left\|b^{+}\right\|+\left\|b^{-}\right\|$. This is called the Jordan decomposition of $b$. Furthermore, the absolute value $|b|$ of $b$ with respect to the polar decomposition of $b$ is equal to $b^{+}+b^{-}$(see Eymard [8], (1.3)).

Let $C_{\rho}^{*}(G)$ be the reduced group $C^{*}$-algebra and let $B_{\rho}(G)$ be the dual of $C_{\rho}^{*}(G)$. Then both $A(G)$ and $B_{\rho}(G)$ are closed ideals of $B(G) . C_{\rho}^{*}(G)=C^{*}(G)$ if and only if $G$ is amenable.

Let $\pi$ be a continuous unitary representation of $G$ on a Hilbert space $H_{\pi}$. For any $\xi, \eta \in H_{\pi}$, the coefficient function defined by $f(x)=\langle\pi(x) \xi, \eta\rangle$ is an element of $B(G)$. We define $A_{\pi}(G)$ to be the norm closure of the subspace of $B(G)$ consisting of all the coefficient functions with respect to the representation $\pi$. If $\rho$ is the left regular representation of $G$ and $\Phi$ is the universal representation of $G$, then $A(G)=A_{\rho}(G)$ and $B(G)=A_{\Phi}(G)$. The following decomposition theorem is an immediate result of Theorem 3.18 and Corollary 3.13 in Arsac [1].

Theorem 2.1. Let $G$ be a locally compact group. Then there is a unique continuous unitary representation $\pi$ of $G$ such that $B(G)=A(G) \oplus A_{\pi}(G)$, and $\rho$ and $\pi$ are disjoint (see [7] or [1] for the notion of disjoint representations).

We will denote $A_{\pi}(G)$ in Theorem 2.1 by $B^{s}(G)$. For each element $b$ in $B(G)$, the decomposition of $b$ in Theorem 2.1 is denoted by $b=b_{u}+b_{s}$, and is called the Lebesgue-type decomposition of $b$, where $b_{u} \in A(G)$ is called an ultraweakly, continuous functional on $C^{*}(G)$ and $b_{s} \in B^{s}(G)$ is called a singular functional on $C^{*}(G)$ i.e., the Lebesgue-type decomposition of $b$ is the following:

$$
b=b_{u}+b_{s} \text { with }\|b\|=\left\|b_{u}\right\|+\left\|b_{s}\right\|, \quad \text { where } b_{u} \in A(G) \text { and } b_{s} \in B^{s}(G) .
$$




\section{Characterizations of $A(G)$ And $B^{s}(G)$}

In this section we will characterize $A(G)$ and $B^{s}(G)$ in terms of some elementary properties of functions on $G$. Corollary 3.8 and Corollary 3.9 are our main results.

For any $f \in L^{1}(G)$ and subset $K$ of $G, \operatorname{supp}(f) \subseteq K^{c}$ means $f 1_{K}=0$ almost everywhere with respect to the Haar measure.

Let $\widetilde{B}(G)$ be the subset of $B(G)$ consisting of all elements $b \in B(G)$ that satisfy the following:

for any $\epsilon>0$, there is a compact subset $K \subset G$ such that $|\langle f, b\rangle|<\epsilon$

for all $f \in L^{1}(G)$ with $\|f\|_{C^{*}(G)} \leq 1$ and $\operatorname{supp}(f) \subset K^{c}$.

Let $\widetilde{\widetilde{B}}(G)$ be the subset of $B(G)$ consisting of all elements $b \in B(G)$ that satisfy the following:

for any $\epsilon>0$ and any compact subset $K \subset G$, there is an $f \in L^{1}(G)$ with $\|f\|_{C^{*}(G)} \leq 1$ and $\operatorname{supp}(f) \subset K^{c}$ such that $|\langle f, b\rangle|>\|b\|-\epsilon$.

We will show in this section that $A(G)=\widetilde{B}(G)$ and $B^{s}(G)=\widetilde{\widetilde{B}}(G)$.

Proposition 3.1. Let $G$ be a locally compact group. Then $A(G) \subseteq \widetilde{B}(G)$.

Proof. This is a special case of Proposition 6.1.

Proposition 3.2. Let $G$ be a locally compact group. Then the canonical mapping $\pi: B^{s}(G) \rightarrow B(G) / A(G)$ is a linear isometry. Consequently, for any $b+A(G) \in$ $B(G) / A(G), b_{s} \in B^{s}(G)$ is the unique element in $b+A(G)$ such that $\left\|b_{s}\right\|=$ $\|b+A(G)\|$, where $b=b_{u}+b_{s}$ is the Lebesgue-type decomposition of $b$.

Proof. It is obvious that $\pi$ is linear. Let $b_{s} \in B^{s}(G)$. Then, for any $a \in A(G)$, $\left\|b_{s}-a\right\|=\left\|b_{s}\right\|+\|a\|$. Hence

$$
\left\|\pi\left(b_{s}\right)\right\|=\inf \left\{\left\|b_{s}-a\right\|: a \in A(G)\right\}=\left\|b_{s}\right\| .
$$

Let $b=b_{u}+b_{s}$ be the Lebesgue-type decomposition of $b$, where $b_{u} \in A(G)$ and $b_{s} \in B^{s}(G)$. Thus, $b_{s} \in b+A(G)$. So $\left\|b_{s}\right\|=\left\|\pi\left(b_{s}\right)\right\|=\|b+A(G)\|$. If $b^{\prime} \in b+A(G)$ with $\left\|b^{\prime}\right\|=\|b+A(G)\|$, then $b_{s}-b^{\prime} \in A(G)$. Hence $b^{\prime}=\left(b^{\prime}-b_{s}\right)+b_{s}$ is the Lebesgue-type decomposition of $b^{\prime}$ by the uniqueness of the decomposition. So $\left\|b^{\prime}\right\|=\left\|b^{\prime}-b_{s}\right\|+\left\|b_{s}\right\|$. But $\left\|b^{\prime}\right\|=\|b+A(G)\|=\left\|b_{s}\right\|$. Therefore $\left\|b^{\prime}-b_{s}\right\|=0$ and so $b^{\prime}=b_{s}$.

Let $K$ be a compact subset of $G$. We denote

$$
A_{K}(G)=\{u \in A(G): \operatorname{supp}(u) \subseteq K\}
$$

The following lemma is contained in the proof of Theorem $B_{1}$ in Granirer and Leinert [11] (p. 464). It plays an important role in the proof of our main result.

Lemma 3.3. Let $G$ be a locally compact group. Then $A_{K}(G)$ is a $\sigma\left(B(G), C^{*}(G)\right)$ closed subspace of $B(G)$.

Let $N$ be a closed normal subgroup of $G$ and let $\pi_{N}: G \rightarrow G / N$ be the canonical map. For any $f \in L^{1}(G), \dot{f}(\dot{x})=\int_{N} f(x \xi) d \xi$ defines a function in $L^{1}(G / N)$, where $\dot{x}=\pi_{N}(x)$ for $x \in G$. Moreover, there is a Haar measure on $G / N$ such that

$$
\int_{G / N}\left\{\int_{N} f(x \xi) d \xi\right\} d \dot{x}=\int_{G} f(x) d x
$$

for all $f \in L^{1}(G)$ (see Reiter [20], pp. 59, 70). 
In addition, if $N$ is compact, then $A(G / N)$ may be identified with the subalgebra of $A(G)$ consisting of the functions which are periodic with respect to $N$. Moreover, $M_{N}: A(G) \rightarrow A(G / N)$, defined by $\left\langle M_{N}(u), \dot{x}\right\rangle=\int_{N} u(x \xi) d \xi, \dot{x}=\pi_{N}(x) \in G / N$, is a Banach space retraction (see Herz [13], p. 106).

To obtain the main results of this paper, we will need an analogous result for $B(G)$ and the technique of its proof to reduce the case to second countable groups.

Proposition 3.4. Let $N$ be a compact subgroup of a locally compact group $G$. If $\pi: G \rightarrow \mathcal{B}\left(H_{\pi}\right)$ is a continuous unitary representation of $G$ on a Hilbert space $H_{\pi}$, then $P \in \mathcal{B}\left(H_{\pi}\right)$, defined by

$$
\langle P \xi, \eta\rangle=\int_{N}\langle\pi(t) \xi, \eta\rangle d t, \quad \xi, \eta \in H_{\pi},
$$

is a self-adjoint projection with $\|P\| \leq 1$. If, in addition, $N$ is a normal subgroup of $G$, then $P H_{\pi}$ is a $\pi(G)$-invariant subspace of $H_{\pi}$. Furthermore, $\dot{\pi}: G / N \rightarrow$ $\mathcal{B}\left(P H_{\pi}\right)$, defined by

$$
\langle\dot{\pi}(\dot{x}) \xi, \eta\rangle=\int_{N}\langle\pi(t x) \xi, \eta\rangle d t, \quad \xi, \eta \in P H_{\pi}
$$

is a continuous unitary representation of $G / N$ on the Hilbert space $P H_{\pi}$ and $\dot{\pi}(\dot{x}) \xi=\pi(x) \xi$ for all $\xi \in P H_{\pi}$.

Proof. It is obvious that $P$ is linear and, for any $\xi, \eta \in H_{\pi}$,

$$
\begin{aligned}
|\langle P \xi, \eta\rangle| & =\left|\int_{N}\langle\pi(t) \xi, \eta\rangle d t\right| \\
& \leq \int_{N}|\langle\pi(t) \xi, \eta\rangle| d t \\
& \leq \int_{N}\|\xi\|\|\eta\| d t \\
& =\|\xi\|\|\eta\| .
\end{aligned}
$$

So $P \in \mathcal{B}\left(H_{\pi}\right)$ with $\|P\| \leq 1$. To prove that $P$ is a self-adjoint projection, for any $\xi, \eta \in H_{\pi}$, we have

$$
\left\langle P^{2} \xi, \eta\right\rangle=\int_{N} \int_{N}\left\langle\pi\left(t_{1} t_{2}\right) \xi, \eta\right\rangle d t_{2} d t_{1}=\int_{N} \int_{N}\left\langle\pi\left(t_{2}\right) \xi, \eta\right\rangle d t_{2} d t_{1}=\langle P \xi, \eta\rangle,
$$

and by a similar calculation using the fact that $N$ is compact and unimodular, we have $\left\langle P^{*} \xi, \eta\right\rangle=\langle P \xi, \eta\rangle$. Hence $P^{2}=P$ and $P^{*}=P$.

Let $N$ be normal. For any $P \xi \in P H_{\pi}$ and $\eta \in H_{\pi}$, we have

$$
\begin{aligned}
\langle P \pi(x) P \xi, \eta\rangle & =\int_{N} \int_{N}\left\langle\pi\left(t_{2}\right) \pi(x) \pi\left(t_{1}\right) \xi, \eta\right\rangle d t_{1} d t_{2} \\
& =\int_{N} \int_{N}\left\langle\pi\left(x\left(x^{-1} t_{2} x\right) t_{1}\right) \xi, \eta\right\rangle d t_{1} d t_{2} \\
& =\int_{N} \int_{N}\left\langle\pi\left(x t_{1}\right) \xi, \eta\right\rangle d t_{1} d t_{2} \\
& =\langle\pi(x) P \xi, \eta\rangle
\end{aligned}
$$

since $N$ is normal and so $x^{-1} t_{2} x \in N$ for all $t_{2} \in N$. Thus, $P \pi(x) P \xi=\pi(x) P \xi$ and $P H_{\pi}$ is $\pi(G)$-invariant. 
Now we show that $\dot{\pi}$ is well defined. In fact, if $\dot{x}=\dot{y}$ in $G / N$ and $\xi, \eta \in P H_{\pi}$, then

$$
\begin{aligned}
\langle\dot{\pi}(\dot{x}) \xi, \eta\rangle & =\langle\dot{\pi}(\dot{x}) P \xi, \eta\rangle \\
& =\int_{N} \int_{N}\left\langle\pi\left(t_{1}\left(x t_{2} y^{-1}\right) y\right) \xi, \eta\right\rangle d t_{1} d t_{2} \\
& =\int_{N} \int_{N}\left\langle\pi\left(t_{1} y\right) \xi, \eta\right\rangle d t_{1} d t_{2} \\
& =\langle\dot{\pi}(\dot{y}) \xi, \eta\rangle
\end{aligned}
$$

since $x t_{2} y^{-1} \in N$ for all $t_{2} \in N$ and $N$ is unimodular. Thus, $\dot{\pi}(\dot{x})=\dot{\pi}(\dot{y})$. If $\xi, \eta \in P H_{\pi}$, then

$$
\begin{aligned}
\langle\dot{\pi}(\dot{x}) \xi, \eta\rangle & =\int_{N}\langle\pi(t x) \xi, \eta\rangle d t \\
& =\int_{N}\left\langle\pi(x) \xi, \pi\left(t^{-1}\right) \eta\right\rangle d t \\
& =\langle\pi(x) \xi, P \eta\rangle=\langle\pi(x) \xi, \eta\rangle .
\end{aligned}
$$

So $\dot{\pi}(\dot{x}) \xi=\pi(x) \xi$. Also, since $P H_{\pi}$ is $\pi(G)$-invariant, $\dot{\pi}(\dot{x}) \xi=\pi(x) \xi \in P H_{\pi}$ for all $\xi \in P H_{\pi}$ and it is clear that $\dot{\pi}(\dot{x})$ is linear and bounded. For any $\dot{x}, \dot{y} \in G / N$,

$$
\dot{\pi}(\dot{x} \dot{y})=\pi(x y)=\pi(x) \pi(y)=\dot{\pi}(\dot{x}) \dot{\pi}(\dot{y}) \text { on } P H_{\pi} .
$$

This shows that $\dot{\pi}$ is a homomorphism. Also,we have

$$
(\dot{\pi}(\dot{x}))^{*}=(\pi(x))^{*}=\pi\left(x^{-1}\right)=\dot{\pi}\left(\dot{x}^{-1}\right) \text { on } P H_{\pi}
$$

So $\dot{\pi}(\dot{x})$ is unitary. For any $S O T$-open set $O$ in $\mathcal{B}\left(P H_{\pi}\right), \dot{\pi}^{-1}(O)$ is open in $G / N$ since $\Pi_{N}^{-1}\left(\dot{\pi}^{-1}(O)\right)=(\pi)^{-1}(O)$ is open in $G$, where $\pi: G \rightarrow \mathcal{B}\left(P H_{\pi}\right)$ (the restriction of $\pi$ onto $P H_{\pi}$ ) is a continuous unitary representation of $G$ and $\Pi_{N}: G \rightarrow$ $G / N$ is the canonical map.

Corollary 3.5. Let $N$ be a compact subgroup of a locally compact group $G$. If $b \in B(G)$, then $b^{N}(x)=\int_{N} b(t x) d t$ defines an element of $B(G)$ with $\left\|b^{N}\right\| \leq\|b\|$ and $b^{N}$ is constant on each right coset of $N$ in $G$. If, in addition, $N$ is a normal subgroup of $G$, then $\dot{b}(\dot{x})=\int_{N} b(x t) d t$ defines an element of $B(G / N)$ with $\|\dot{b}\| \leq$ $\|b\|$. Furthermore, $\dot{b}=b^{N}$ if we consider $B(G / N)$ as a subspace of $B(G)$ (see Eymard [8], (2.26)).

Proof. Let $b(x)=\langle\pi(x) \xi, \eta\rangle$ for a continuous unitary representation $\left(\pi, H_{\pi}\right)$ of $G$, where $H_{\pi}$ is a Hilbert space and $\xi, \eta \in H_{\pi}$ with $\|b\|=\|\xi\|\|\eta\|$ (see Eymard [8], (2.14)). Then

$$
b^{N}(x)=\int_{N}\langle\pi(t x) \xi, \eta\rangle d t=\langle P \pi(x) \xi, \eta\rangle=\langle\pi(x) \xi, P \eta\rangle, \quad x \in G,
$$

where $P \in \mathcal{B}\left(H_{\pi}\right)$ defined in Proposition 3.4 is a self-adjoint projection with $\|P\| \leq$ 1. Hence $b^{N} \in B(G)$ and $\left\|b^{N}\right\| \leq\|\xi\|\|P \eta\| \leq\|b\|$.

Let $N$ be normal. Then, for any $x \in G$,

$$
\begin{aligned}
\int_{N} \int_{N} b\left(t_{1} x t_{2}\right) d t_{1} d t_{2} & =\int_{N} \int_{N} b\left(x\left(x^{-1} t_{1} x\right) t_{2}\right) d t_{2} d t_{1} \\
& =\int_{N} \int_{N} b\left(x t_{2}\right) d t_{2} d t_{1}=\dot{b}(x)
\end{aligned}
$$


since $N$ is normal and $x^{-1} t_{1} x \in N$ for all $t_{1} \in N$. On the other hand, since $x^{-1} t_{2} x \in N$ for all $t_{2} \in N$ and $N$ is unimodular, we have

$$
\begin{aligned}
\int_{N} \int_{N} b\left(t_{1} x t_{2}\right) d t_{1} d t_{2} & =\int_{N} \int_{N} b\left(t_{1}\left(x t_{2} x^{-1}\right) x\right) d t_{1} d t_{2} \\
& =\int_{N} \int_{N} b\left(t_{1} x\right) d t_{1} d t_{2}=b^{N}(x) .
\end{aligned}
$$

Hence $b^{N}(x)=\dot{b}(x)$ for all $x \in G$.

Let $\left(\dot{\pi}, P H_{\pi}\right)$ be the continuous unitary representation of $G / N$ as in Proposition 3.4. Hence we have

$$
\begin{aligned}
\langle\dot{\pi}(\dot{x}) P \xi, P \eta\rangle & =\langle\pi(x) P \xi, P \eta\rangle \\
& =\int_{N} \int_{N}\left\langle\pi(x) \pi\left(t_{1}\right) \xi, \pi\left(t_{2}\right) \eta\right\rangle d t_{2} d t_{1} \\
& =\int_{N} \int_{N}\left\langle\pi\left(x\left(x^{-1} t_{2}^{-1} x\right) t_{1}\right) \xi, \eta\right\rangle d t_{2} d t_{1} \\
& =\int_{N} \int_{N}\left\langle\pi\left(x t_{1}\right) \xi, \eta\right\rangle d t_{1} d t_{2} \\
& =\int_{N} b\left(x t_{1}\right) d t_{1} \\
& =\dot{b}(\dot{x})
\end{aligned}
$$

since $x^{-1} t_{2}{ }^{-1} x \in N$ for all $t_{2} \in N$. Therefore, $\dot{b} \in B(G / N)$ and $\|\dot{b}\| \leq\|P \xi\|\|P \eta\| \leq$ $\|\xi\|\|\eta\|=\|b\|$ (see Eymard [8], (2.14)).

Corollary 3.6. Let $N$ be a compact normal subgroup of a locally compact group $G$ and let $M_{N}: B(G) \rightarrow B(G / N)$ be the retraction defined in Corollary 3.5 by $M_{N}(b)=\dot{b}$. If we regard $L^{1}(G / N)$ as a subspace of $L^{1}(G)$ consisting of periodic functions on $G$ with respect to $N$, then, for any $f \in L^{1}(G / N) \subseteq B(G / N)^{*}$, $M_{N}^{*}(f)=f \in L^{1}(G) \subseteq B(G)^{*}$ and $\|f\|_{C^{*}(G)}=\|f\|_{C^{*}(G / N)}$.

Proof. For any $b \in B(G)$, we have

$$
\begin{aligned}
\left\langle M_{N}^{*}(f), b\right\rangle & =\left\langle f, M_{N}(b)\right\rangle \\
& =\int_{G / N} f(\dot{x}) \dot{b}(\dot{x}) d \dot{x} \\
& =\int_{G / N}\left\{\int_{N} f(x \xi) b(x \xi) d \xi\right\} d \dot{x} \\
& =\int_{G} f(x) b(x) d x \\
& =\langle f, b\rangle
\end{aligned}
$$

since $f$ is constant on each coset of $N$ in $G$. Therefore, $M_{N}^{*}(f)=f$.

By the proof of (2.26) in Eymard [8], $\|f\|_{C^{*}(G / N)} \leq\|f\|_{C^{*}(G)}$. Also, by Corollary 3.5 ,

$$
\|f\|_{C^{*}(G)}=\left\|M_{N}^{*}(f)\right\|_{C^{*}(G)} \leq\left\|M_{N}^{*}\right\|\|f\|_{C^{*}(G / N)} \leq\|f\|_{C^{*}(G / N)} .
$$

Therefore $\|f\|_{C^{*}(G)}=\|f\|_{C^{*}(G / N)}$.

Now we are ready to prove our main theorem. 
Theorem 3.7. Let $G$ be a locally compact group. Then $B^{s}(G) \subseteq \widetilde{\widetilde{B}}(G)$.

Proof. If $G$ is compact, then $B^{s}(G)=\{0\}$. Hence $B^{s}(G) \subseteq \widetilde{\widetilde{B}}(G)$. Suppose $G$ is noncompact. Let $b \in B^{s}(G)$ be with $\|b\|=1$. To show that $b \in \widetilde{\widetilde{B}}(G)$, let a compact subset $K$ of $G$ and $\epsilon>0$ be given. Since $\|b-a\|=\|b\|+\|a\| \geq 1$ for any $a \in A(G)$, by the Hahn-Banach theorem, there is an $F \in B(G)^{*}$ such that $\|F\| \leq 1,\langle F, b\rangle=1$ and $\langle F, a\rangle=0$ for all $a \in A(G)$. Since $B^{*}(G)=\left(C^{*}(G)\right)^{* *}$, and $L^{1}(G)$ is norm dense in $C^{*}(G)$, by Goldstine's theorem, there is a net $f_{\alpha} \in L^{1}(G)$ in the unit ball of $\left(C^{*}(G)\right)^{* *}$ such that $f_{\alpha} \rightarrow F$ in the $\sigma\left(B^{*}(G), B(G)\right)$-topology. Hence $\left\langle f_{\alpha}, a\right\rangle \rightarrow\langle F, a\rangle=0$ for all $a \in A(G)$ and $\left\langle f_{\alpha}, b\right\rangle \rightarrow\langle F, b\rangle=1$. Let $c_{\alpha}$ be a complex number with absolute value 1 and $\left|\left\langle f_{\alpha}, b\right\rangle\right|=\left\langle c_{\alpha} f_{\alpha}, b\right\rangle$. So $\left\langle c_{\alpha} f_{\alpha}, a\right\rangle \rightarrow 0$ for all $a \in A(G),\left\langle c_{\alpha} f_{\alpha}, b\right\rangle \rightarrow 1,\left\langle c_{\alpha} f_{\alpha}, b\right\rangle \geq 0$ and $\left\|c_{\alpha} f_{\alpha}\right\|_{C^{*}(G)}=\left\|f_{\alpha}\right\|_{C^{*}(G)} \leq 1$. We assume, without loss of generality, that $\left\langle f_{\alpha}, b\right\rangle$ is real and $\left\langle f_{\alpha}, b\right\rangle \geq 1-\epsilon$ for all $\alpha$.

Choose an open-closed $\sigma$-compact subgroup $G_{0}$ of $G$ such that $K \subseteq G_{0}$. For each $n>0$, let $V_{n}=\left\{x \in G:\left\|_{x} b-b\right\|+\left\|b_{x}-b\right\|<\frac{1}{n}\right\}$. Since the left and right translations of $b$ define continuous functions from $G$ to $B(G)$ (this follows from the fact that $b(t)=\langle\pi(t) \xi, \eta\rangle$ for some continuous unitary representation $\pi$ and $\|b\| \leq\|\xi\|\|\eta\|), V_{n}$ is an open neighborhood of $e$. There is a compact normal subgroup $N$ of $G_{0}$ such that $N \subset G_{0} \cap\left(\bigcap_{n=1}^{\infty} V_{n}\right)$ and $G_{0} / N$ is second countable (see Hewitt and Ross [14], (8.7), p. 71). Hence $b$ is a periodic function on $G$ with respect to $N$.

Next we will show that we can assume $f_{\alpha}$ is constant on each right coset of $N$ in $G$. For each $\alpha$, we assume, without loss of generality, that $f_{\alpha}$ is continuous with compact support. First, we show that $f_{\alpha}^{N}(x)=\int_{N} f_{\alpha}(t x) d t$ defines an element of $L^{1}(G)$ with the properties that

$$
\left\|f_{\alpha}^{N}\right\|_{C^{*}(G)} \leq 1, \quad\left\langle f_{\alpha}^{N}, b\right\rangle \geq 1-\epsilon \quad \text { and } \quad\left\langle f_{\alpha}^{N}, a\right\rangle \rightarrow 0
$$

for all $a \in A\left(G_{0} / N\right)$, and $f_{\alpha}^{N}$ is constant on each right coset of $N$ in $G$. In fact, it is clear that $f_{\alpha}^{N} \in L^{1}(G)$ (see [20], (4.8)) and $f_{\alpha}^{N}$ is constant on each right coset of $N$ in $G$. For any $u \in B(G)$, we have,

$$
\begin{aligned}
\left\langle f_{\alpha}^{N}, u\right\rangle & =\int_{G} \int_{N} f_{\alpha}(t x) u(x) d t d x \\
& =\int_{N} \int_{G} f_{\alpha}(t x) u(x) d x d t \quad \text { (by Fubini's theorem, see [20], (4.8)) } \\
& =\int_{N} \int_{G} f_{\alpha}(x) u\left(t^{-1} x\right) d x d t \\
& =\int_{G} \int_{N} f_{\alpha}(x) u\left(t^{-1} x\right) d t d x \quad \text { (by Fubini's theorem) } \\
& \left.=\int_{G} \int_{N} f_{\alpha}(x) u(t x) d t d x \quad \text { (by the compactness of } N\right) \\
& =\left\langle f_{\alpha}, u^{N}\right\rangle .
\end{aligned}
$$


Hence $\left|\left\langle f_{\alpha}^{N}, u\right\rangle\right| \leq\left\|f_{\alpha}\right\|_{C^{*}(G)}\left\|u^{N}\right\| \leq\|u\|$ by Corollary 3.5. It follows that $\left\|f_{\alpha}^{N}\right\|_{C^{*}(G)} \leq 1$. Also,

$$
\left\langle f_{\alpha}^{N}, b\right\rangle=\left\langle f_{\alpha}, b^{N}\right\rangle=\left\langle f_{\alpha}, b\right\rangle \geq 1-\epsilon \text { and }\left\langle f_{\alpha}^{N}, a\right\rangle=\left\langle f_{\alpha}, a^{N}\right\rangle=\left\langle f_{\alpha}, a\right\rangle \rightarrow 0
$$

for all $a \in A\left(G_{0} / N\right) \subseteq A(G)$ since both $a, b \in B(G)$ are constant on each right coset of $N$ in $G$ (so $a^{N}=a$ and $b^{N}=b$ ).

Thus, taking $f_{\alpha}^{N}$ if necessary, we may assume, without loss of generality, that $f_{\alpha}$ is constant on each right coset of $N$ in $G$.

Choose $a_{K} \in A\left(G_{0} / N\right)$ such that $a_{K}=1$ on the compact set $K$ and $\operatorname{supp}\left(a_{K}\right)$ denoted by $K_{s}$ is a compact subset of $G_{0} / N$. Then $a_{K} \in A(G)$ since $A\left(G_{0} / N\right) \subseteq$ $A\left(G_{0}\right)$.

Since $G_{0} / N$ is second countable, $L^{2}\left(G_{0} / N\right)$ is norm separable. So $A\left(G_{0} / N\right)$ is separable in norm by the definition of $A\left(G_{0} / N\right)$. Hence, the fact of $\left\langle f_{\alpha}, a\right\rangle \rightarrow 0$ for all $a \in A\left(G_{0} / N\right)$ and $\left\|f_{\alpha}\right\| \leq 1$ implies that there is a sequence $\left\{f_{\alpha_{n}}\right\}$ of $\left\{f_{\alpha}\right\}$ such that $\left\langle f_{\alpha_{n}}, a\right\rangle \rightarrow 0$ for all $a \in A\left(G_{0} / N\right)$. In fact, since $A\left(G_{0} / N\right)$ is separable, the weak*-topology on the unit ball of $A\left(G_{0} / N\right)^{*}$ is metrizable. $f_{\alpha} \rightarrow 0$ in the weak*topology on the unit ball of $A\left(G_{0} / N\right)^{*}$ implies that there exists a sequence $\left\{f_{\alpha_{n}}\right\}$ of $\left\{f_{\alpha}\right\}$ such that $f_{\alpha_{n}} \rightarrow 0$ in the weak*-topology on the unit ball of $A\left(G_{0} / N\right)^{*}$. Hence $\left\langle f_{\alpha_{n}}, a\right\rangle \rightarrow 0$ for all $a \in A\left(G_{0} / N\right)$.

Let $\Omega$ be the unit ball of $A_{K_{s}}\left(G_{0} / N\right)$. Since $K_{s}$ is a compact subset of $G_{0} / N$, $\Omega$ is compact in the $\sigma\left(B\left(G_{0} / N\right), C^{*}\left(G_{0} / N\right)\right)$-topology by Lemma 3.3. For each $n$, by Corollary 3.6, $f_{\alpha_{n}} 1_{G_{0}} \in L^{1}\left(G_{0} / N\right)$ is a $\sigma\left(B\left(G_{0} / N\right), C^{*}\left(G_{0} / N\right)\right)$-continuous function defined on $\Omega$. Also,

$$
\left|\left\langle f_{\alpha_{n}} 1_{G_{0}}, a\right\rangle\right|=\left|\left\langle f_{\alpha_{n}}, a\right\rangle\right| \leq 1, \quad \text { for all } a \in \Omega .
$$

Hence, $\left\|f_{\alpha_{n}} 1_{G_{0}}\right\|_{C(\Omega)} \leq 1$. Thus, the fact of $f_{\alpha_{n}} 1_{G_{0}} \rightarrow 0$ pointwisely on $\Omega$ implies that $f_{\alpha_{n}} 1_{G_{0}} \rightarrow 0$ weakly in $C(\Omega)$ (see Diestel [5], p. 66). Therefore there are positive numbers $\beta_{1}, \beta_{2}, \ldots, \beta_{m}$ and $\alpha_{n_{1}}, \alpha_{n_{2}}, \ldots, \alpha_{n_{m}}$ such that

$$
\sum_{i=1}^{m} \beta_{i}=1 \quad \text { and } \quad\left\|\sum_{i=1}^{m} \beta_{i} f_{\alpha_{n_{i}}} 1_{G_{0}}\right\|_{C(\Omega)}<\epsilon\left\|a_{K}\right\|^{-1} .
$$

Let $g=a_{K} \sum_{i=1}^{m} \beta_{i} f_{\alpha_{n_{i}}} 1_{G_{0}}$. Then, since $a_{K}=1$ on $K, g=\sum_{i=1}^{m} \beta_{i} f_{\alpha_{n_{i}}} 1_{G_{0}}$ on $K$. Furthermore, $\|g\|_{C^{*}(G)} \leq \epsilon$. In fact, for any $u \in B(G)$ with $\|u\| \leq 1$, since $N$ is a normal subgroup of $G_{0}$ and $a_{K} u \in A\left(G_{0}\right) \subseteq B\left(G_{0}\right)$, by Corollary 3.5 , we have

$$
\left(a_{K} u\right)=\left(a_{K} u\right)^{N}=a_{K} u^{N} \in A_{K_{s}}\left(G_{0} / N\right) .
$$

By the previous calculation in this proof, $g^{N}=g$ and $\left\|u^{N}\right\| \leq\|u\| \leq 1$, we have

$$
\begin{aligned}
|\langle g, u\rangle| & =\left|\left\langle g^{N}, u\right\rangle\right|=\left|\left\langle g, u^{N}\right\rangle\right| \\
& =\left|\left\langle\sum_{i=1}^{m} \beta_{i} f_{\alpha_{n_{i}}} 1_{G_{0}}, a_{K} u^{N}\right\rangle\right| \\
& \leq\left\|\sum_{i=1}^{m} \beta_{i} f_{\alpha_{n_{i}}} 1_{G_{0}}\right\|_{C(\Omega)} \cdot\left\|a_{K} u^{N}\right\|<\epsilon .
\end{aligned}
$$

Let $h=\sum_{i=1}^{m} \beta_{i} f_{\alpha_{n_{i}}}$. Then $\|h\|_{C^{*}(G)} \leq \sum_{i=1}^{m} \beta_{i}\left\|f_{\alpha_{n_{i}}}\right\|_{C^{*}(G)} \leq 1$. Set $f=h-g$. Then 
(1) since $g=h$ on $K, \operatorname{supp}(f) \subseteq K^{c}$;

(2) $\|f\|_{C^{*}(G)} \leq\|h\|_{C^{*}(G)}+\|g\|_{C^{*}(G)} \leq 1+\epsilon$;

(3) since $|\langle g, b\rangle| \leq\|g\|_{C^{*}(G)}\|b\|<\epsilon$, we have the following

$$
|\langle f, b\rangle| \geq|\langle h, b\rangle|-|\langle g, b\rangle|=\left\langle\sum_{i=1}^{m} \beta_{i} f_{\alpha_{n_{i}}}, b\right\rangle-|\langle g, b\rangle|>1-2 \epsilon .
$$

Therefore, $b \in \widetilde{\widetilde{B}}(G)$ and $B^{s}(G) \subseteq \widetilde{\widetilde{B}}(G)$.

Corollary 3.8. Let $G$ be a locally compact group. Then $B^{s}(G)=\widetilde{\widetilde{B}}(G)$.

Proof. We will show that $\widetilde{\widetilde{B}}(G) \subseteq B^{s}(G)$. Let $b \in \widetilde{\widetilde{B}}(G)$. We will show that $\|b\|=\|b+A(G)\|$. For any $a \in \bar{A}(G)$, by Proposition 3.1, $a \in \widetilde{B}(G)$. Hence, for any $\epsilon>0$, there is a compact subset $K$ of $G$ such that for any $f \in L^{1}(G)$ with $\|f\|_{C^{*}(G)} \leq 1$ and $\operatorname{supp}(f) \subseteq K^{c}$ we have $|\langle f, a\rangle|<\epsilon$. Since $b \in \widetilde{\widetilde{B}}(G)$, there is an $f \in L^{1}(G)$ with $\|f\|_{C^{*}(G)} \leq 1$ and $\operatorname{supp}(f) \subseteq K^{c}$ such that $|\langle f, b\rangle|>\|b\|-\epsilon$. So $|\langle f, a\rangle|<\epsilon$ for this $f$ and

$$
\|b-a\| \geq|\langle f, b-a\rangle| \geq|\langle f, b\rangle|-|\langle f, a\rangle|>\|b\|-2 \epsilon .
$$

Hence $\|b-a\| \geq\|b\|$, and $\|b\|=\inf \{\|b-a\|: a \in A(G)\}=\|b+A(G)\|$. It follows from Proposition 3.2 that $b=b_{s}$. Therefore $b \in B^{s}(G)$. By Theorem 3.7, $B^{s}(G)=\widetilde{\widetilde{B}}(G)$.

Corollary 3.9. Let $G$ be a locally compact group. Then $A(G)=\widetilde{B}(G)$.

Proof. We show that $\widetilde{B}(G) \subseteq A(G)$. Let $b \in \widetilde{B}(G)$. Suppose $b=b_{u}+b_{s}$ is the Lebesgue-type decomposition of $b$. Then $\|b\|=\left\|b_{u}\right\|+\left\|b_{s}\right\|$, and $b_{u} \in A(G)$ and $b_{s} \in B^{s}(G)$. It is obvious from the definition that $\widetilde{B}(G)$ is a subspace of $B(G)$. By Proposition 3.1, $b_{u} \in \widetilde{B}(G)$. Hence $b_{s}=b-b_{u} \in \widetilde{B}(G)$. For any $\epsilon>0$, there is a compact subset $K$ of $G$ such that $\left|\left\langle f, b-b_{u}\right\rangle\right|<\epsilon$ for all $f \in L^{1}(G)$ with $\|f\|_{C^{*}(G)} \leq 1$ and $\operatorname{supp}(f) \subset K^{c}$. It follows from Theorem 3.7 that $b_{s}=b-b_{u} \in$ $B^{s}(G) \subseteq \widetilde{\widetilde{B}}(G)$. So there is an $f \in L^{1}(G)$ with $\|f\|_{C^{*}(G)} \leq 1$ and $\operatorname{supp}(f) \subset K^{c}$ such that

$$
\left|\left\langle f, b-b_{u}\right\rangle\right|=\left|\left\langle f, b_{s}\right\rangle\right|>\left\|b_{s}\right\|-\epsilon=\left\|b-b_{u}\right\|-\epsilon .
$$

Hence, $\left\|b-b_{u}\right\|<2 \epsilon$. Since $\epsilon$ is arbitrary, $b=b_{u}$ is in $A(G)$. Therefore, $\widetilde{B}(G) \subseteq$ $A(G)$ and $\widetilde{B}(G)=A(G)$ by Proposition 3.1.

It is known that (see Pier [19], p. 209) when $G$ is amenable, for any $b \in B(G)$,

$$
\|b\|=\sup \{\|a b\|: a \in A(G),\|a\| \leq 1\} .
$$

We will characterize $A(G)$ and $B^{s}(G)$ in terms of the multiplier norm condition above.

Theorem 3.10. Let $G$ be an amenable locally compact group and let $b \in B(G)$. Then

(1) $b \in A(G)$ if and only if $b$ satisfies the following: for any $\epsilon>0$, there exists a compact subset $K$ of $G$ such that

$$
\sup \left\{\|a b\|: a \in A(G),\|a\| \leq 1 \text { and } \operatorname{supp}(a) \subseteq K^{c}\right\}<\epsilon .
$$


(2) $b \in B^{s}(G)$ if and only if $b$ satisfies the following: for any compact subset $K$ of $G$,

$$
\|b\|=\sup \left\{\|a b\|: a \in A(G),\|a\| \leq 1 \text { and } \operatorname{supp}(a) \subseteq K^{c}\right\} .
$$

Proof. (1) Let $b \in A(G)$ and $\epsilon>0$ be given. Since the set of the elements of $A(G)$ with compact support is dense in $A(G)$, there is an $a_{K} \in A(G)$ with compact support $K$ such that $\left\|b-a_{K}\right\|<\frac{1}{2} \epsilon$. So for any $a \in A(G)$ with $\|a\| \leq 1$ and $\operatorname{supp}(a) \subseteq K^{c}$, we have $\|a b\|=\left\|a b-a a_{K}\right\| \leq\|a\|\left\|b-a_{K}\right\|<\frac{1}{2} \epsilon$. Therefore, $(*)$ is satisfied.

Conversely, let $b$ satisfy $(*)$ and $\|b\|=1$. Let $\epsilon>0$ be given. Then there is a compact subset $K$ of $G$ satisfying (*). Since $G$ is amenable, there is an $a_{K} \in A(G)$ such that $a_{K}=1$ on $K,\left\|a_{K}\right\|<2$. By the multiplier norm condition above, there is an $a \in A(G)$ with $\|a\| \leq 1$ and $\left\|a b\left(1-a_{K}\right)\right\|>\left\|b\left(1-a_{K}\right)\right\|-\epsilon$. Since $a\left(1-a_{K}\right) \in A(G)$ and $\operatorname{supp}\left(a\left(1-a_{K}\right)\right) \subseteq K^{c}$, by $(*)$, we have

$$
\left\|b-a_{K} b\right\|<\left\|a b\left(1-a_{K}\right)\right\|+\epsilon \leq\left\|a\left(1-a_{K}\right)\right\| \epsilon+\epsilon \leq 4 \epsilon .
$$

Note that $a_{K} b \in A(G)$, so $b$ is a norm limit of elements in $A(G)$. Therefore $b \in A(G)$.

(2) Suppose $b \in B^{s}(G)$ and $\|b\|=1$. Let a compact subset $K$ of $G$ be given. For any $\epsilon>0$, by Corollary 3.8, there is an $f \in L^{1}(G)$ such that $\operatorname{supp}(f)$ is compact, $\|f\|_{C^{*}(G)} \leq 1$ and $|\langle f, b\rangle|>1-\epsilon$. Since $G$ is amenable, there is an $a_{K} \in A(G)$ with the properties $a_{K}=1$ on $K \bigcup(\operatorname{supp}(f))$ and $\left\|a_{K}\right\|<1+\epsilon$. So

$$
\left\|a_{K} b\right\| \geq\left|\left\langle f, a_{K} b\right\rangle\right|=|\langle f, b\rangle|>1-\epsilon .
$$

Since $b \in B^{s}(G)$ and $a_{K} b \in A(G),\left\|b-a_{K} b\right\|=\|b\|+\left\|a_{K} b\right\|>2-\epsilon$ by the norm condition of the Lebesgue-type decomposition of $b-a_{K} b$. Hence there is an $a \in A(G)$ with $\|a\| \leq 1$ such that $\left\|a\left(b-a_{K} b\right)\right\|>2-\epsilon$ by the multiplier norm condition above. Let $u=\frac{1}{2} a\left(1-a_{K}\right)$. Then $\|u\| \leq 1+\frac{1}{2} \epsilon, \operatorname{supp}(u) \subseteq K^{c}$ and $\|u b\|=\left\|\frac{1}{2} a\left(1-a_{K}\right) b\right\|>1-\frac{1}{2} \epsilon$. Therefore

$$
\|b\|=\sup \left\{\|a b\|: a \in A(G),\|a\| \leq 1 \text { and } \operatorname{supp}(a) \subseteq K^{c}\right\} .
$$

Conversely, let b satisfy that for any compact subset $K$ of $G$,

$$
\|b\|=\sup \left\{\|a b\|: a \in A(G),\|a\| \leq 1 \text { and } \operatorname{supp}(a) \subseteq K^{c}\right\} .
$$

Suppose $\|b\|=1$ without loss of generality. Then for any compact subset $K$ of $G$ and any $\epsilon>0$, there is an $a \in A(G)$ with $\|a\| \leq 1, \operatorname{supp}(a) \subseteq K^{c}$ and $\|a b\|>1-\epsilon$. By the definition of the norm of $a b$ in $A(G)$, there is an $f \in L^{1}(G)$ with $\|f\|_{C^{*}(G)} \leq 1$ and $|\langle f, a b\rangle| \geq 1-\epsilon$. So $a f \in L^{1}(G)$ with $\|a f\|_{C^{*}(G)} \leq 1$ such that $|\langle a f, b\rangle|>1-\epsilon$ and $\operatorname{supp}(a f) \subseteq K^{c}$. Thus $b \in \widetilde{\widetilde{B}}(G)$. Therefore $b \in B^{s}(G)$ by Corollary 3.8.

\section{Properties of $B^{s}(G)$}

In this section we will investigate the basic properties of $B^{s}(G)$. Let $M(G)$ be the space of all bounded measures on $G$. The convolution invariant property by elements of $M(G)$ follows from [1]. Then we will apply our characterization theorems proved in section 3 to study some other properties of $B^{s}(G)$. We will show that $b \in B(G)$ is positive definite if and only if both $b_{u}$ and $b_{s}$ are positive definite, and $b \in B(G)$ is hermitian if and only if both $b_{u}$ and $b_{s}$ are hermitian. Consequently, we have that if $b \in B^{s}(G)$, then $\tilde{b} \in B^{s}(G)$ and if $b \in B^{s}(G)$ is hermitian, then $b^{+}, b^{-}$and $|b|$ are all in $B^{s}(G)$, where $b=b^{+}-b^{-}$is the Jordan 
decomposition of $b_{s}$. We will also prove that $B^{s}(G)$ is invariant with respect to subgroups and quotient groups. Then we will use this property to show that $B^{s}(G)$ may not be a subalgebra of $B(G)$ even when $G$ is abelian.

Let $M$ be a von Neumann algebra and let $M_{*}$ be its predual. Then $M_{*}$ has the left [right] $M$-module structure: if $T \in M$ and $u \in M_{*}, T . u[u . T]$ is an element in $M_{*}$ defined by, for any $S \in M,\langle T . u, S\rangle=\langle u, S T\rangle[\langle u . T, S\rangle=\langle u, T S\rangle]$.

Let $\pi$ be a continuous unitary representation of $G$ on a Hilbert space $H_{\pi} . V N_{\pi}(G)$ will denote the von Neumann algebra generated by $\pi(G)$ in $\mathcal{B}\left(H_{\pi}\right)$. Then the predual of $V N_{\pi}(G)$ is $A_{\pi}(G)$ (see [1], p. 13). If $\mu \in M(G)$ and $a \in A_{\pi}(G)$, then $\mu . a=a *(\Delta \check{\mu})$ and $a . \mu=\check{\mu} * a$ (see Proposition 2.8 in [1]), where $\check{\mu}$ is defined by $\int_{G} f(x) d \check{\mu}=\int_{G} f\left(x^{-1}\right) d \mu$ for each continuous function $f$ on $G$ with compact support.

Theorem 4.1. Let $G$ be a locally compact group. For any $T \in V N_{\pi}(G), T . A_{\pi}(G)$ $\subseteq A_{\pi}(G)$ and $A_{\pi}(G) . T \subseteq A_{\pi}(G)$. In particular, $A_{\pi}(G)$ is invariant under translations by elements of $G$ and invariant under convolution by the elements of $M(G)$ from both left and right.

Proof. This follows from Proposition 3.16 and Proposition 2.8 in [1].

Corollary 4.2. Let $G$ be a locally compact group. Then both $A(G)$ and $B^{s}(G)$ are invariant under translations by elements of $G$ and invariant under convolution by the elements of $M(G)$ from both left and right.

Proof. This is an immediate consequence of Theorem 4.1 and the facts that $A(G)=$ $A_{\rho}(G)$ and $B^{s}(G)=A_{\pi}(G)$, where $\rho$ is the left regular representation of $G$ and $\pi$ is a continuous unitary representation of $G$ (see Theorem 2.1).

Theorem 4.3. Let $G$ be a locally compact group. Let $b \in B(G)$ and $b=b_{u}+b_{s}$ be the Lebesgue-type decomposition of $b$. Then $b$ is positive definite if and only if both $b_{u}$ and $b_{s}$ are positive definite.

Proof. If both $b_{u}$ and $b_{s}$ are positive definite, it is obvious that $b=b_{u}+b_{s}$ is also positive definite.

Conversely, let $b$ be positive definite. Then $b$ is a positive functional on $C^{*}(G)$. Let $\left\{f_{\lambda}\right\}$ be a bounded approximate identity in $C^{*}(G)$ (see Theorem 3.1.1 and Theorem 3.3.3 in Murphy [16]). Then

$$
\left\|b_{u}\right\|+\left\|b_{s}\right\|=\|b\|=\lim _{\lambda}\left\langle b, f_{\lambda}\right\rangle=\lim _{\lambda}\left\langle b_{u}, f_{\lambda}\right\rangle+\lim _{\lambda}\left\langle b_{s}, f_{\lambda}\right\rangle .
$$

Since $\lim _{\lambda}\left\langle b_{u}, f_{\lambda}\right\rangle \leq\left\|b_{u}\right\|$ and $\lim _{\lambda}\left\langle b_{s}, f_{\lambda}\right\rangle \leq\left\|b_{s}\right\|, \lim _{\lambda}\left\langle b_{u}, f_{\lambda}\right\rangle=\left\|b_{u}\right\|$ and $\lim _{\lambda}\left\langle b_{s}, f_{\lambda}\right\rangle=\left\|b_{s}\right\|$. Therefore, both $b_{u}$ and $b_{s}$ are positive functional on $C^{*}(G)$ (see Theorem 3.3.3 in Murphy [16]), so both are positive definite.

Corollary 4.4. Let $G$ be a locally compact group. Suppose $b \in B^{s}(G)$ is hermitian. If $b=b^{+}-b^{-}$is the Jordan decomposition of $b$, where $b^{+}$and $b^{-}$are positive definite with $\|b\|=b^{+}(e)+b^{-}(e)$, then $b^{+} \in B^{s}(G)$ and $b^{-} \in B^{s}(G)$. Consequently, $|b| \in B^{s}(G)$.

Proof. Suppose $b \in B^{s}(G)$ is hermitian and $b=b^{+}-b^{-}$is the Jordon decomposition of $b$ (see Eymard [8], (1.3)). Let

$$
b^{+}=\left(b^{+}\right)_{u}+\left(b^{+}\right)_{s} \text { and } b^{-}=\left(b^{-}\right)_{u}+\left(b^{-}\right)_{s}
$$


be the Lebesgue-type decompositions of $b^{+}$and $b^{-}$, respectively. Then $\left(b^{+}\right)_{u},\left(b^{-}\right)_{u}$ $\in A(G)$ and $\left(b^{+}\right)_{s},\left(b^{-}\right)_{s} \in B^{s}(G)$ are positive definite by Theorem 4.3. By the uniqueness of the decomposition,

$$
b=\left(\left(b^{+}\right)_{u}-\left(b^{-}\right)_{u}\right)+\left(\left(b^{+}\right)_{s}-\left(b^{-}\right)_{s}\right)
$$

is the Lebesgue-type decomposition of $b$.

Since $b \in B^{s}(G)$, by the uniqueness of the Lebesgue-type decomposition of $b$ again, $\left(b^{+}\right)_{u}=\left(b^{-}\right)_{u}$. So $b=\left(b^{+}\right)_{s}-\left(b^{-}\right)_{s}$. Hence,

$$
\begin{aligned}
\left(b^{+}\right)_{s}(e)+\left(b^{-}\right)_{s}(e) & =\left\|\left(b^{+}\right)_{s}\right\|+\left\|\left(b^{-}\right)_{s}\right\| \\
& \geq\|b\|=b^{+}(e)+b^{-}(e) \\
& =\left(b^{+}\right)_{u}(e)+\left(b^{+}\right)_{s}(e)+\left(b^{-}\right)_{u}(e)+\left(b^{-}\right)_{s}(e)
\end{aligned}
$$

implies

$$
\left\|\left(b^{+}\right)_{u}\right\|+\left\|\left(b^{-}\right)_{u}\right\|=\left(b^{+}\right)_{u}(e)+\left(b^{-}\right)_{u}(e)=0 .
$$

Therefore $\left(b^{+}\right)_{u}=\left(b^{-}\right)_{u}=0$, and so $b^{+}=\left(b^{+}\right)_{s} \in B^{s}(G)$ and $b^{-}=\left(b^{-}\right)_{s} \in B^{s}(G)$. Consequently, $|u|=b^{+}+b^{-}$(see Eymard [8], (1.3)) is in $B^{s}(G)$.

Corollary 4.5. Let $G$ be a locally compact group. Suppose $b \in B(G)$ is positive definite. Then $b$ is a singular functional (i.e., $b \in B^{s}(G)$ ) if and only if there are no $a \in A(G)$ such that $0<a \leq b$ as functionals on $C^{*}(G)$.

Proof. Let $b \in B^{s}(G)$ be singular. Suppose there is an $a \in A(G)$ such that $0 \leq a \leq b$ as functionals on $C^{*}(G)$. Since $b-a=(-a)+(b)$ is the Lebesgue-type decomposition of $b-a$, we have $\|b-a\|=\|b\|+\|a\|$. On the other hand, since $a, b$ and $b-a$ are positive definite,

$$
\|b\|=b(e)=(b(e)-a(e))+a(e)=\|b-a\|+\|a\|=\|b\|+2\|a\| .
$$

Hence $\|a\|=0$ and $a=0$.

Conversely, suppose there are no $a \in A(G)$ such that $0<a \leq b$ as functionals on $C^{*}(G)$. Let $b=b_{u}+b_{s}$ be the Lebesgue-type decomposition of $b$. Then, by Theorem 4.3 , both $b_{u}$ and $b_{s}$ are positive definite. So $0 \leq b_{u} \leq b$ as functionals on $C^{*}(G)$. Thus, $b_{u}=0$. Therefore $b=b_{s} \in B^{s}(G)$.

Corollary 4.6. Let $G$ be a locally compact group. Then $b \in B(G)$ is a singular functional if and only if $b$ is a finite linear combination of singular positive definite elements of $B(G)$.

Proof. Each bounded linear functional $b$ on $C^{*}(G)$ is a finite linear combination $\sum \alpha_{i} b_{i}$ of states $b_{i}$ on $C^{*}(G)$. Then

$$
b=\sum \alpha_{i}\left(b_{i}\right)_{u}+\sum \alpha_{i}\left(b_{i}\right)_{s}, \text { where } b_{i}=\left(b_{i}\right)_{u}+\left(b_{i}\right)_{s} \text { for each } i .
$$

If $b$ is singular, then $b=\sum \alpha_{i}\left(b_{i}\right)_{s}$ by the uniqueness of the decomposition. By Theorem 4.3 , each $\left(b_{i}\right)_{s}$ is positive definite.

Conversely, since $B^{s}(G)$ is a subspace, a finite linear combination of singular functionals on $C^{*}(G)$ is itself a singular functional on $C^{*}(G)$.

Proposition 4.7. Let $G$ be a locally compact group. If $b \in B^{s}(G)$, then $\tilde{b} \in B^{s}(G)$. Furthermore, if $b \in B(G)$ and $b=b_{u}+b_{s}$ is the Lebesgue-type decomposition of $b$, then so is $\tilde{b}=\widetilde{\left(b_{u}\right)}+\widetilde{\left(b_{s}\right)}$. Consequently, we have that $b$ is hermitian if and only if both $b_{u}$ and $b_{s}$ are hermitian. 
Proof. Let $b$ be singular. By Corollary $3.8, b \in \widetilde{\widetilde{B}}(G)$. We will show that $\tilde{b}$ is also singular. Let $K \subseteq G$ be compact and $\epsilon>0$. Then there is an $f \in L^{1}(G)$ with $\operatorname{supp}(f) \subseteq\left(K^{-1}\right)^{c}$ and $\|f\|_{C^{*}(G)} \| \leq 1$ such that $|\langle b, f\rangle|>\|b\|-\epsilon$. So $f^{*} \in L^{1}(G)$ with $\left\|f^{*}\right\|_{C^{*}(G)}=\|f\|_{C^{*}(G)} \leq 1$ and $\operatorname{supp}\left(f^{*}\right) \subseteq K^{c}$ such that $\left|\left\langle\tilde{b}, f^{*}\right\rangle\right|=|\langle b, f\rangle| \geq$ $\|b\|-\epsilon=\|\tilde{b}\|-\epsilon$ since $\|b\|=\|\tilde{b}\|$ (see Eymard [8], (2.15)). Hence, $\tilde{b} \in B^{s}(G)$ by Corollary 3.8.

Let $b=b_{u}+b_{s}$ be the Lebesgue-type decomposition of $b$. Then $\tilde{b}=\widetilde{\left(b_{u}\right)}+\widetilde{\left(b_{s}\right)}$. It is clear that $\widetilde{\left(b_{u}\right)} \in A(G)$ by the definition of $A(G)$ and also $\widetilde{\left(b_{s}\right)} \in B^{s}(G)$ with $\|\tilde{b}\|=\|b\|=\left\|b_{u}\right\|+\left\|b_{s}\right\|=\left\|\widetilde{\left(b_{u}\right)}\right\|+\left\|\widetilde{\left(b_{s}\right)}\right\|$. By the uniqueness of the decomposition, $\tilde{b}=\widetilde{\left(b_{u}\right)}+\widetilde{\left(b_{s}\right)}$ is the Lebesgue-type decomposition of $\tilde{b}$.

If $b$ is hermitian, then $b=\tilde{b}$. So by the uniqueness of the Lebesgue-type decomposition of $b, b_{u}=\widehat{\left(b_{u}\right)}$ and $b_{s}=\widehat{\left(b_{s}\right)}$. Therefore, both $b_{u}$ and $b_{s}$ are hermitian. It is clear that if both $b_{u}$ and $b_{s}$ are hermitian, then so is $b=b_{u}+b_{s}$.

Now we show that $B^{s}(G)$ is invariant with respect to the subgroups and the quotient groups.

Let $N$ be an open subgroup of a locally compact group $G$. By (2.31) of Eymard [8], if $G$ is amenable, then the restriction $b \rightarrow b \mid N$ is a surjective homomorphism of the Banach algebra $B(G)$ onto $B(N)$. If $b \in B^{s}(G)$, then the restriction $b \mid N \in$ $B(N)$ may not be in $B^{s}(N)$. In fact, if $N$ is a compact open subgroup of a noncompact locally compact group $G$, then $B^{s}(N)=\{0\}$. So $1_{G} \mid N=1_{N} \notin B^{s}(N)$. But $1_{G} \in B^{s}(G)$ (see Theorem 5.3).

Let $N$ be a subgroup of $G$. For each function $f$ on $N, f^{\circ}$ denotes a function defined on $G$ which is $f$ on $N$ and 0 on $G \sim N$.

Proposition 4.8. Let $G$ be an amenable locally compact group. If $N$ is an open subgroup of $G$, then $b \rightarrow b^{\circ}$ is a linear isometry from $B(N)$ into $B(G)$. Furthermore, if $b \in B^{s}(N)$, then $b^{\circ} \in B^{s}(G)$.

Proof. Since $G$ is amenable, $N$ is amenable and $A(N)$ has a bounded approximate identity $\left\{a_{i}\right\}$ such that $\left\|a_{i}\right\| \rightarrow 1$. Thus, $a_{i} b \rightarrow b$ in the $\sigma\left(B(N), C^{*}(N)\right)$-topology for all $b \in B(N)$. Since $A(N)$ is identified with a subalgebra of $A(G)$ (see Herz [13], Proposition 5) and $a_{i} b \in A(N) \subseteq B(G), b^{\circ}=\lim _{i} a_{i} b$ in the $\sigma\left(B(G), C^{*}(G)\right)$ topology and $b^{\circ}$ is in $B(G)$ for all $b \in B(N)$. Furthermore, we have

$$
\begin{aligned}
\|b\|_{B(N)} & \leq \varlimsup_{i}\left\|a_{i} b\right\|_{B(N)}=\varlimsup_{i}\left\|a_{i} b\right\|_{A(N)} \\
& =\varlimsup_{i}\left\|a_{i} b\right\|_{A(G)}=\varlimsup_{i}\left\|a_{i} b^{\circ}\right\|_{A(G)} \leq\left\|b^{\circ}\right\|_{B(G)} .
\end{aligned}
$$

On the other hand, by (2.31) in Eymard [8],

$$
\left\|b^{\circ}\right\|_{B(G)} \leq\left\|\left.b^{\circ}\right|_{N}\right\|_{B(N)}=\|b\|_{B(N)} .
$$

So $\left\|b^{\circ}\right\|_{B(G)}=\|b\|_{B(N)}$. It is clear that $b \rightarrow b^{\circ}$ is linear. Therefore it is a linear isometry.

Suppose $b \in B^{s}(N)$ with $\|b\|=1$. Let $\epsilon>0$ and a compact subset $K$ of $G$ be given. Then $N \cap K$ is a compact subset of $N$. Since $b \in B^{s}(N)$, by Corollary 3.8, there is an $f \in L^{1}(N)$ such that $\operatorname{supp}(f) \subseteq(N \cap K)^{c},\|f\| \leq 1$ and $|\langle f, b\rangle|>1-\epsilon$. Hence $f^{\circ} \in L^{1}(G)$ such that $\operatorname{supp}\left(f^{\circ}\right) \subseteq K^{c},\left\|f^{\circ}\right\|_{C^{*}(G)}=\|f\|_{C^{*}(N)} \leq 1$ (see Eymard [8], (2.31)) and $\left|\left\langle f^{\circ}, b^{\circ}\right\rangle\right|=|\langle f, b\rangle|>1-\epsilon$. So $b^{\circ} \in B^{s}(G)$. 
The following example shows that $B^{s}(G)$ may not be a subalgebra of $B(G)$ even when $G$ is abelian (also see Hewitt and Ross [14], (19.21) and (19.26)). It is known that $A(G)$ is an ideal of $B(G)$.

Example 4.9. Let $G$ be an amenable discrete group with two infinite cyclic subgroups $G_{a}$ and $G_{b}$, where $a$ and $b$ are generators of $G_{a}$ and $G_{b}$, respectively. Suppose $G_{a} \cap G_{b}=\{e\}$. Then $1_{G_{a}} \in B^{s}\left(G_{a}\right)$ and $1_{G_{b}} \in B^{s}\left(G_{b}\right)$ (see Theorem 5.3) imply $1_{G_{a}}^{\circ}, 1_{G_{b}}^{\circ} \in B^{s}(G)$ by Proposition 4.8. But $1_{G_{a}}^{\circ} 1_{G_{b}}^{\circ}=1_{\{e\}} \notin B^{s}(G)$. So $B^{s}(G)$ is not a subalgebra of $B(G)$.

Let $N$ be a closed normal subgroup of $G$. Then $B(G / N)$ is identified with a subspace of $B(G)$ consisting of functions which are constant on equivalence classes modulo $N$ (see Eymard [8], (2.26)).

Proposition 4.10. If $N$ is a compact normal subgroup of $G$, then $B^{s}(G / N)$ is identified with a subspace of $B^{s}(G)$ consisting of functions in $B^{s}(G)$ which are constant on equivalence classes modulo $N$.

Proof. Suppose $b \in B^{s}(G / N)$ with $\|b\|=1$. Let $\epsilon>0$ and a compact subset $K$ of $G$ be given. Then $\pi_{N}(K)$ is a compact subset of $G / N$, where $\pi_{N}: G \rightarrow G / N$ is the canonical map. Since $b \in B^{s}(G / N)$, by Corollary 3.8, there is an $f \in L^{1}(G / N)$ such that $\operatorname{supp}(f) \subseteq\left(\pi_{N}(K)\right)^{c},\|f\| \leq 1$ and $|\langle f, b\rangle|>1-\epsilon$. Hence $f \in L^{1}(G)$ such that $\operatorname{supp}(f) \subseteq(K N)^{c} \subseteq K^{c},\|f\|_{C^{*}(G)}=\|f\|_{C^{*}(G / N)} \leq 1$ (by Corollary 3.6) and $|\langle f, b\rangle|>1-\epsilon$. So $b \in B^{s}(G)$ by Corollary 3.8 .

\section{Some applichtions}

In this section we will have two applications. The Banach space $X$ is said to have the Dunford-Pettis property (DPP for short) if, for any Banach space $Y$, every weakly compact linear operator $u: X \rightarrow Y$ sends weak Cauchy sequences into norm convergent sequences. See Diestel [6] for more information on this property. Lau and Ülger [15] (Theorem 4.2) proved that $B(G)$ has the $D P P$ if and only if $G$ is a Moore group (see Palmer [17] for Moore groups). We extend this result to $B_{\rho}(G)$.

Proposition 5.1. Let $G$ be a locally compact group. Then $A(G)$ is complemented in $B_{\rho}(G)$ and in $B(G)$.

Proof. This is an immediate consequence of Theorem 2.1 since $A(G) \subseteq B_{\rho}(G)$.

Theorem 5.2. Let $G$ be a locally compact group. Then the following are equivalent:

(1) $G \in[M O O R E]$.

(2) $B(G)$ has the DPP.

(3) $A(G)$ has the DPP.

(4) $B_{\rho}(G)$ has the DPP.

Proof. (1) $\Longleftrightarrow(2)$ This is proved in Theorem 4.2 of Lau and Ülger [15].

$(1) \Longleftrightarrow(3)$ It follows from Bunce [3] and Theorem 4.2 of Lau and Ülger [15] that $A(G)$ has the DPP if and only if $V N(G)$ is Type I finite if and only if $G \in$ [MOORE].

$(1) \Longrightarrow(4)$ Let $G \in[\mathrm{MOORE}]$. Then $G$ is amenable (see Palmer [17], p. 298). So $B_{\rho}(G)=B(G)$ has the DPP.

$(4) \Longrightarrow(3)$ If $B_{\rho}(G)$ has the DPP, then $A(G)$ has the DPP since the subspace $A(G)$ is complemented in $B_{\rho}(G)$ by Proposition 5.1 (see Diestel [6]). 
Theorem 5.3. Let $G$ be a locally compact group. If $G$ is noncompact, then $1 \in$ $B^{s}(G)$. Consequently, $\|1+a\|=1+\|a\|$ for all $a \in A(G)$.

Proof. Suppose that $x_{n} \in G$ and $x_{n} \rightarrow \infty$. Let $1=b_{u}+b_{s}$ be the Lebesgue-type decomposition of $1 \in B(G)$. Then

$$
1=\left\langle 1, \delta_{x_{n}}\right\rangle=\left\langle b_{u}, \delta_{x_{n}}\right\rangle+\left\langle b_{s}, \delta_{x_{n}}\right\rangle .
$$

Since $\left\langle b_{u}, \delta_{x_{n}}\right\rangle \rightarrow 0$ as $n \rightarrow \infty$, we have $\left\langle b_{s}, \delta_{x_{n}}\right\rangle \rightarrow 1$ as $n \rightarrow \infty$. Hence $\left\|b_{s}\right\| \geq 1$. Since $\|1\|=\langle 1, e\rangle=1$ (see Eymard [8], (2.6)) and $\|1\|=\left\|b_{u}\right\|+\left\|b_{s}\right\|,\left\|b_{u}\right\|=0$. Therefore $b_{u}=0$ and $1 \in B^{s}(G)$. So $\|1+a\|=1+\|a\|$ for all $a \in A(G)$.

Remarks 5.4. (a) If $G$ is compact, then obviously this is not true.

(b) Let $G$ be a noncompact amenable locally compact group. If $K$ is a compact subset of $G$ and $\epsilon>0$, then it is known that there is an $a_{K} \in A(G)$ such that $a_{K}=1$ on $K,\left\|a_{K}\right\|<1+\epsilon$ and supp $\left(a_{K}\right)$ is compact. By our Theorem 5.3, we have the following estimation of the norm of $1-a_{K}$ :

$$
2 \leq\left\|1-a_{K}\right\|=1+\left\|a_{K}\right\|<2+\epsilon .
$$

(c) If $G$ is abelian and noncompact, then the dual group $\hat{G}$ is not discrete. This theorem means that $\left\|\delta_{\hat{e}}+f\right\|_{M(\hat{G})}=1+\|f\|_{1}$ for all $f \in L^{1}(\hat{G})$ where $\hat{e}$ is the unit of $\hat{G}$.

\section{THE CASE OF $p \neq 2$}

If we have a $p$-version decomposition theorem as Theorem 2.1, then the proofs of our main results in this paper work for $p \neq 2$. So there would be a $p$-version for each of our main results in this paper (see section 3). But we do not know whether this $p$-version decomposition theorem is true. In this section, we will prove a $p$-version characterization theorem as Corollary 3.9 for amenable locally compact groups.

The Figà-Talamanca-Herz algebra $A_{p}(G)$ is the space of functions $u: G \rightarrow C$ which can be represented, nonuniquely, as

$$
u=\sum_{n=1}^{\infty} v_{n} * \check{u}_{n} \quad \text { for } \quad u_{n} \in L^{p}(G) \text { and } \quad v_{n} \in L^{q}(G) \text { with } \sum_{n=1}^{\infty}\left\|u_{n}\right\|_{p}\left\|v_{n}\right\|_{q}<\infty
$$

and $\|u\|=\inf \sum_{n=1}^{\infty}\left\|u_{n}\right\|_{p}\left\|v_{n}\right\|_{q}$, where the infimum is taken over all possible representations of $u$ and $\frac{1}{p}+\frac{1}{q}=1$. It is known that $A_{p}(G)$ is a regular tauberian algebra under the pointwise multiplication, $A_{2}(G)=A(G)$ and $A_{p}(G)$ has a bounded approximate identity if and only if $G$ is amenable. Each $f \in L^{1}(G)$ defines a bounded linear functional on $A_{p}(G)$ by

$$
\langle f, u\rangle=\int_{G} f(x) u(x) d x \quad \text { for } \quad u \in A_{p}(G) .
$$

The norm of $f$ as an element of $A_{p}(G)^{*}$ and the operator norm of $f$ as an element of $\mathcal{B}\left(L^{p}(G)\right)$ are the same; that is,

$$
\|f\|_{A_{p}(G)}=\sup _{u \in A_{p}(G),\|u\| \leq 1}|\langle f, u\rangle| \quad \text { and } \quad\|f\|_{\mathcal{B}\left(L^{p}(G)\right)}=\sup _{g \in L^{p}(G),\|g\|_{p} \leq 1}\|f * g\|_{p}
$$


are equal. It follows that $L^{1}(G)$ is a subspace of $A_{p}(G)^{*}$. Let $P F_{p}(G)$ be the closure of $L^{1}(G)$ in $A_{p}(G)^{*}$ with respect to the norm topology. When $p=2, P F_{2}(G)$ is $C_{\rho}^{*}(G)$, the reduced group $C^{*}$-algebra of $G$.

$B_{p}(G)$ is the pointwise multiplier algebra of $A_{p}(G)$, consisting of the continuous functions $v$ on $G$ such that $u v \in A_{p}(G)$ for all $u \in A_{p}(G)$. The norm on $B_{p}(G)$ is defined by

$$
\|v\|_{B_{p}(G)}=\sup \left\{\|v u\|_{A_{p}(G)} \text { and }\|u\|_{A_{p}(G)} \leq 1\right\} .
$$

Observe that $A_{p}(G) \subseteq B_{p}(G)$ and if $v \in A_{p}(G),\|v\|_{B_{p}(G)} \leq\|v\|_{A_{p}(G)}$. It has been shown that $P F_{p}(G)^{*}=B_{p}(G)$ if and only if $G$ is amenable (see Cowling [4]), in this case, for any $b \in B_{p}(G), b \in P F_{p}(G)^{*}$ is defined by $\langle b, f\rangle=\int_{G} b(t) f(t) d t$ for $f \in L^{1}(G)$ and $B_{2}(G)=B(G)$.

Let $\widetilde{B}_{p}(G)$ be the subset of $B_{p}(G)$ consisting of all elements $b \in B_{p}(G)$ that satisfy the following statement: For any $\epsilon>0$, there is a compact subset $K \subset G$ such that $|\langle f, b\rangle|<\epsilon$ for all $f \in L^{1}(G)$ with $\|f\|_{P F_{p}(G)} \leq 1$ and $\operatorname{supp}(f) \subseteq K^{c}$.

Proposition 6.1. Let $G$ be a locally compact group. Then $A_{p}(G) \subseteq \widetilde{B}_{p}(G)$.

Proof. Suppose that $a \in A_{p}(G)$ and let $\epsilon>0$ be given. Since the subspace of $L^{p}(G)$ (or $L^{q}(G)$ ) consisting of the elements with compact support is dense in $L^{p}(G)$ (or $\left.L^{q}(G)\right)$, by the definition of $A_{p}(G),\left\|a-\sum_{i=1}^{n} \check{v}_{i} * u_{i}\right\|<\epsilon$ for some $u_{i} \in L^{p}(G)$ and $v_{i} \in L^{q}(G)$ with compact support $(i=1,2, \ldots, n)$. Let $K$ be the support of $\sum_{i=1}^{n} \check{v}_{i} * u_{i}$. Then $K$ is compact. For any $f \in L^{1}(G)$ with $\|f\|_{P F_{p}(G)} \leq 1$ and $\operatorname{supp}(f) \subset K^{c}$, we have

$$
|\langle f, a\rangle|=\left|\left\langle f, a-\sum_{i=1}^{n} \check{v}_{i} * u_{i}\right\rangle\right| \leq\left\|a-\sum_{i=1}^{n} \check{v}_{i} * u_{i}\right\|<\epsilon .
$$

Therefore, $a \in \widetilde{B}_{p}(G)$.

Corollary 6.2. Let $G$ be an amenable locally compact group. Then $A_{p}(G)=$ $\widetilde{B}_{p}(G)$.

Proof. We only show that $\widetilde{B}_{p}(G) \subseteq A_{p}(G)$. Let $b \in \widetilde{B}_{p}(G)$. For any $\epsilon>0$, there is a compact subset $K$ of $G$ such that $|\langle f, b\rangle|<\epsilon$ for all $f \in L^{1}(G)$ with $\|f\|_{P F_{p}(G)} \leq 1$ and $\operatorname{supp}(f) \subset K^{c}$. Since $G$ is amenable, there is an $a \in A_{p}(G)$ with $a=1$ on $K$ and $\|a\| \leq 2$. For any $f \in L^{1}(G)$ with $\|f\|_{P F_{p}(G)} \leq 1$, then $f-a f \in L^{1}(G)$ with $\|f-a f\|_{P F_{p}(G)} \leq 3$. Also, $\operatorname{supp}(f-a f) \subseteq K^{c}$. Hence, $|\langle b-a b, f\rangle|=|\langle b, f-a f\rangle| \leq 3 \epsilon$. Hence $\|b-a b\| \leq 3 \epsilon$. It follows from the facts that $a b \in A_{p}(G)$ and $A_{p}(G)$ is norm closed in $B_{p}(G)$ that $b \in A_{p}(G)$. Therefore, $\widetilde{B}_{p}(G) \subseteq A_{p}(G)$ and $\widetilde{B}_{p}(G)=A_{p}(G)$ by Proposition 6.1.

\section{REFERENCES}

1. G. Arsac, Sur l'espace de Banach engendré par les coefficients d'une représentation unitaire, Publ. Dép. Math. (Lyon) 13 (1976), 1-101. MR 56:3180

2. L. Baggett and K. Taylor, Groups with completely reducible regular representation, Proc. Amer. Math. Soc. 72 (1978), 593-600. MR 80b:22009

3. L.J. Bunce, The Dunford-Pettis property in the predual of a Von Neumann algebra, Proc. Amer. Math. Soc. 116 (1992), 99-100. MR 92k:46100 
4. M. Cowling, An application of Littlewood-Paley theory in harmonic analysis, Math. Ann. 241 (1979), 83-96. MR 81f: 43003

5. J. Diestel, Sequences and series in Banach spaces, Graduate Texts in Math., Springer-Verlag, New York, 1984. MR 85i:46020

6. 15-60. MR 82i:46023

7. J. Dixmier, $C^{*}$-Algebras, North-Holland, New York, 1977. MR 56:16388

8. P. Eymard, L'algèbre de Fourier d'un groupe localement compact, Bull. Soc. Math. France, 92 (1964), 181-236. MR 37:4208

9. V. Flory, On the Fourier algebra of a locally compact amenable group, Proc. Amer. Math. Soc. 29 (1971), 603-606. MR 44:371

10. __ Eine Lebesgue-Zerlegung und funktorielle Eigenschaften der Fourier-Stieltjes Algebra, Inaugural Dissertation, University of Heidelberg (1972).

11. E. E. Granirer and M. Leinert, On some topologies which coincide on the unit sphere of the Fourier-Stieltjes algebra $B(G)$ and of the measure algebra $M(G)$, Rocky Mountain J Math. 11 (1981), 459-472. MR 85f:43009

12. F.P. Greenleaf, Invariant Means on Topological Groups and their Applications, Van Nostrand Reinhold, New York, 1969. MR 40:4776

13. C. Herz, Harmonic synthesis for subgroups, Ann. Inst. Fourier (Grenoble) 23 (1973), 91-123. MR 50:7956

14. E. Hewitt and K. Ross, Abstract harmonic analysis, Vol. I, Second Edition, Springer-Verlag, Berlin, 1979. MR 81k:43001

15. A.T. Lau and A. Ülger, Some geometric properties on the Fourier and Fourier Stieltjes algebras of locally compact groups, Arens regularity and related problems, Trans. Amer. Math. Soc. 337 (1993), 321-359. MR 93j:22007

16. G. J. Murphy, $C^{*}$-algebra and operator theory, Academic Press, 1990. MR 91m:46084

17. T.W. Palmer, Classes of nonabelian, noncompact locally compact groups, Rocky Mountain J Math. 8 (1978), 683-741. MR 81j:22003

18. A.L.T. Paterson, Amenability, Mathematical Surveys and Monographs, 29, Amer. Math. Soc., Providence, Rhode Island, 1988. MR 90e:43001

19. J.P. Pier, Amenable Locally Compact Groups, Wiley, New York, 1984. MR 86a:43001

20. H. Reiter, Classical Harmonic Analysis and Locally Compact Groups, Oxford, Clarendon, 1968. MR 46:5933

21. K. Taylor, Geometry of Fourier algebras and locally compact groups with atomic representations, Math. Ann. 262 (1983), 183-190. MR 84h:43020

Department of Mathematical Sciences, Lakehead University, Thunder Bay, Ontario P7E 5E1 CANADA

E-mail address: tmiao@thunder.lakeheadu.ca 